\title{
BIFURCATION AND COMPLEX PHENOMENA IN CHAOTIC SYSTEMS COUPLED BY TRANSMISSION LINE
}

\author{
Junji Kawata ${ }^{\dagger}$, Yoshifumi Nishio ${ }^{\ddagger, *}$ and Akio Ushida \\ $\dagger$ Tokushima Bunri University, Kagawa, JAPAN \\ $\ddagger$ Tokushima University, Tokushima, JAPAN \\ * Swiss Federal Institute of Technology, Lausanne, Switzerland
}

\begin{abstract}
In this study, bifurcation and complex phenomena in synchronizing chaotic oscillators coupled by a lossless transmission line are investigated. The period-doubling bifurcation with varying the time delay is observed in our circuit model. Further the voltage distribution of transmission line is simulated in order to investigate whether the current flowing through the transmission line is constant or not. It is found that the subsystems synchronize although the current through the transmission line keeps on varying.
\end{abstract}

\section{INTRODUCTION}

Since synchronization of chaos has been reported by Pecora et al. [1], it has received a great deal of attention. A large number of recent papers have been published in this area. However, almost studies on chaos synchronization reported so far, have dealt with synchronization phenomena observed from chaotic oscillators coupled by lumped elements as in [2]. We think that the investigation of systems with timedelayed signal is very important, because there exists the time delay of signals in the natural fields and real systems utilizing chaos will be affected by the time delay.

A few studies on systems coupled by elements with time delay have been reported. In [3] two chaotic systems coupled by two delay lines are investigated. It has been shown via numerical experiments that two chaotic circuits synchronized when the time delay exists.

We have also reported synchronization phenomena in a chaotic system coupled by transmission line [4], [5]. It is confirmed by simulations and experiments that the subsystems synchronize by adjusting the characteristic impedance of the line when the time delay increases.

Further, in this paper, we present some new results. The bifurcation diagrams with respect to the time delay are shown. The period-doubling bifurcation is observed from $v_{C 1}$-coupled system. The voltage distribution of the transmission line is also given in order to investigate whether the current through the line is varying or not. It is found that the current flowing through the coupling transmission line keeps on varying, which is different from the case of lumped element coupling.

\section{CIRCUIT MODEL}

Fig. 1 shows the circuit models used in this paper. In these models, the Chua's circuit is used as each chaotic subcircuit and two subcircuits are coupled by a transmission line. The Chua's circuit is a extremely simple system but it exhibits the complex dynamics of bifurcation and chaos.

In our simulations, the transmission line is modeled using the method of characteristics, where the transmission line is replaced by the characteristic model [6]. After normalizing, we obtain the following circuit equations.

$v_{C 1}$-coupled system:

$$
\begin{aligned}
& \dot{x}_{k}=\alpha\left(y_{k}-x_{k}-f\left(x_{k}\right)+\gamma\left(w_{k}-x_{k}\right)\right), \\
& \dot{y}_{k}=x_{k}-y_{k}+z_{k}, \\
& \dot{z}_{k}=-\beta y_{k}
\end{aligned}
$$

$v_{C 2}$-coupled system:

$$
\begin{aligned}
& \dot{x}_{k}=\alpha\left(y_{k}-x_{k}-f\left(x_{k}\right)\right) \\
& \dot{y}_{k}=x_{k}-y_{k}+z_{k}+\gamma\left(w_{k}-y_{k}\right), \\
& \dot{z}_{k}=-\beta y_{k}
\end{aligned}
$$

Where $k=1,2$,

$$
f(x)=b x+\frac{1}{2}(a-b)[|x+1|-|x-1|],
$$

and

$$
\begin{aligned}
& \alpha=C_{2} / C_{1}, \quad \beta=C_{2} / L G^{2}, \gamma=Y_{0} / G, \\
& \hat{t}=G t / C_{2}, \hat{\tau}=G \tau / C_{2}, \cdots \cdot,=d / d \hat{t}, \\
& x_{k}=v_{C 1 k} / B_{p}, \quad y_{k}=v_{C 2 k} / B_{p}, \quad z_{k}=i_{L k} / G B_{p}, \\
& w_{k}=e_{k}(\hat{t}-\hat{\tau}) / B_{p}, \quad a=m_{0} / G, \quad b=m_{1} / G .
\end{aligned}
$$

$Y_{0}$ and $\tau$ are the characteristic admittance and time delay of the transmission line respectively, and $e_{k}$ is the waveform generator of the characteristic model to simulate the reflection. 


\section{NUMERICAL RESULTS}

The following has been confirmed numerically and experimentally in our previous studies [4], [5].

- The subsystems synchronize when the time delay exists.

- When only the time delay increases, oscillations of the subsystems stop for $v_{C 1}$-coupled system and the trajectories of subsystems diverge for $v_{C 2}$-coupled system.

- By adjusting the characteristic impedance, the subsystems in both models become to synchronize when the time delay increases.

\subsection{Bifurcation Diagram}

As mentioned above, since the chaotic subsystems stop oscillation or their orbits diverge according to the time delay, it is natural to think that the systems may give rise to bifurcation. Thus we plot bifurcation diagrams with respect to the time delay. The circuit parameters are fixed as follows:

$$
\begin{aligned}
& \alpha=10, \quad a=-1.2, \quad b=-0.75, \quad B_{p}=1, \\
& x_{1}=1.0, \quad y_{1}=0.02, \quad z_{1}=0.0, \\
& x_{2}=-0.7, \quad y_{2}=0.4, \quad z_{2}=0.08 .
\end{aligned}
$$

Figs. 3 and 4 show the bifurcation diagrams. Where the horizontal and vertical axes correspond to the time delay and voltage $v_{C 1}$, respectively. Note that the two subsystems are synchronized completely for the parameter values in these figures (except the range of divergence). Surprisingly enough, for $v_{C 1}$-coupled system, rich bifurcation phenomena including the period-doubling route to chaos and several periodic windows are observed with decreasing the dime delay even if the two subsystems are synchronized completely. In [3] it has been also reported that their circuit model exhibits the period-doubling bifurcation under certain conditions. However, their subsystems did not synchronize completely during the bifurcation route.

On the other hand, in $v_{C 2}$-coupled system, chaos synchronization can be observed for relatively wide range of time delay, since periodic orbits do not emerge even if the time delay becomes fairly large. By increasing the time delay further, the trajectories eventually diverge. Moreover, many types of periodic window take place according as values of $\hat{\tau}$.

\subsection{Voltage Distribution}

At first thought, we expected that the current through the transmission line may be zero or constant, because in systems coupled by a linear resistor the current flowing the coupling resistor is zero or constant. Thus we investigate the current distribution of the transmission line.
Let the current and voltage of transmission line be $i(x, \hat{t})$ and $v(x, \hat{t})$, respectively. Since $i(x, \hat{t})=v(x, \hat{t}) / Z_{0}$, we can investigate the voltage distribution. In our simulations, the transmission line is divided to 50 segments of transmission lines, and then each of them are replaced with the characteristic models.

The results with the subsystems synchronizing are shown in Figs. 5 and 6. In turn from left, the attractor in the left subcircuit, the attractor in the right one, the state of synchronization, and the voltage distribution are given. In the figure of voltage distribution, the axis toward the right direction is the distance from the left end of transmission line, the upward axis is the voltage $v_{C 1}$ or $v_{C 2}$, and axis toward the depth corresponds to normalized time $\hat{t}$.

As you can see from this figure, the voltage distribution is not constant for both systems. So we can conclude that in our systems the current is flowing through the transmission line, which is different to the case of lumped element coupling.

\section{CONCLUSION}

In this study, we have reported very interesting phenomena in a chaotic system coupled by a lossless transmission line. Chaos synchronization is also achieved in such system as well as systems coupled with lumped elements. It is found that in our models bifurcation phenomena occurs with variation of the time delay. Also the current flowing through the coupling transmission line keeps on varying, which is different from the case of lumped element coupling.

Hereafter we intend to investigate the systems more fully (e.g. by 2 parameter bifurcation diagrams and so on) and then analyze the systems qualitatively. The systems may have more exciting phenomena according to the characteristic impedance and time delay.

\section{REFERENCES}

[1] L. M. Pecora and T. L. Carroll, "Synchronization in chaotic systems,” Phys. Rev. Lett., vol. 64, no. 4, pp. 821-824, 1990.

[2] L. O. Chua et al., "Chaos synchronization in Chua's circuit," J. Circuits, Syst., Comput., vol. 3, no. 1, pp. 93-108, 1993.

[3] R. Koike et al., "Synchronization of two chaotic circuits coupled by delay line," Proc. of ECCTD'97, vol. 3, pp. 12801285, 1997.

[4] J. Kawata, Y. Nishio and A. Ushida, "Chaos synchronization by transmission line coupling," Proc. of NOLTA'96, pp. 109112, 1996.

[5] J. Kawata, Y. Nishio and A. Ushida, "On synchronization phenomena in chaotic system coupled by transmission line", Proc. IEEE ISCAS'2000, vol. 3, pp. 479-482, 2000.

[6] F. H. Branin, Jr., "Transient analysis of lossless transmission line," Proc. IEEE, vol. 55, pp. 2012-2013, Nov. 1967.

[7] M. P. Kennedy, "Robust OP amp realization of Chua's circuit," Frequenz, vol. 46, pp. 66-80, 1992. 


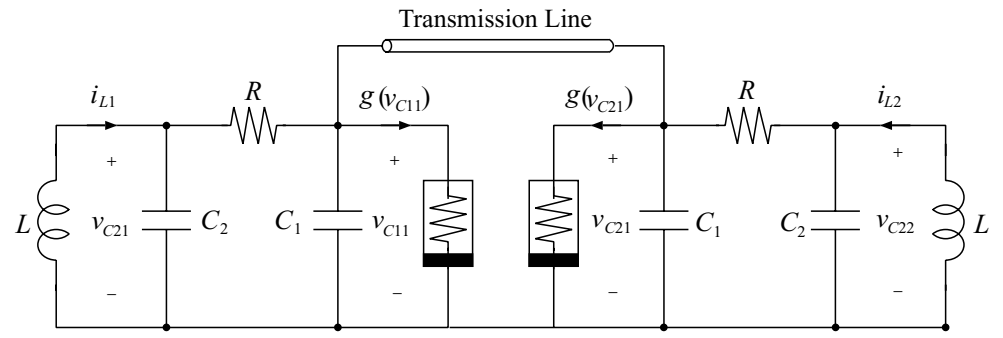

(a)

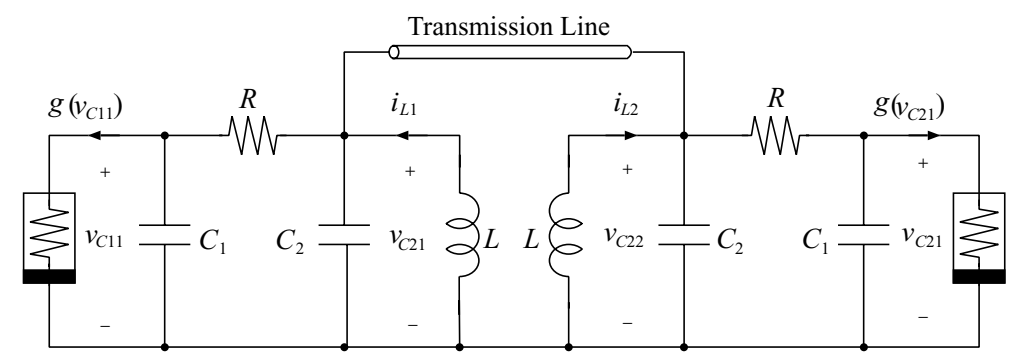

(b)

Figure 1: The circuit model. (a) $v_{C 1}$-coupled system. (b) $v_{C 2}$-coupled system.

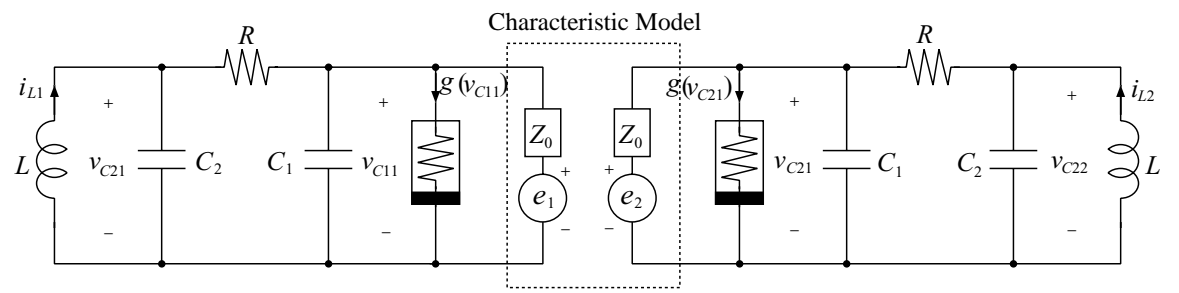

(a)

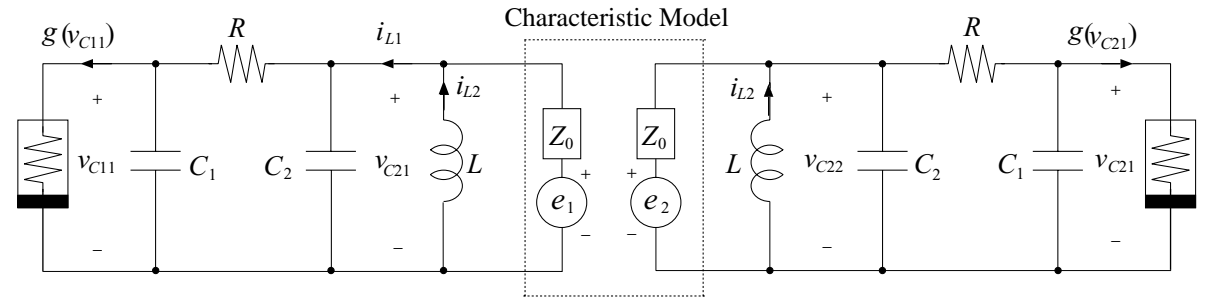

(b)

Figure 2: The equivalent circuits for (a) $v_{C 1}$-coupled system and (b) $v_{C 2}$-coupled system.

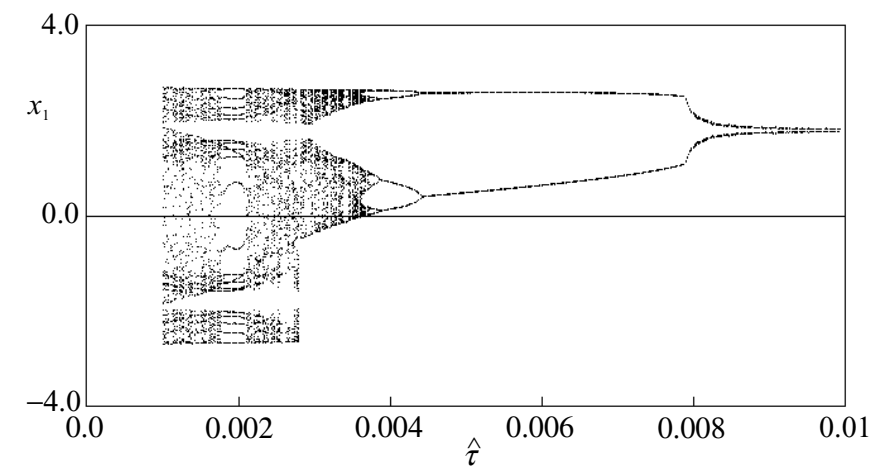

Figure 3: One parameter bifurcation diagram for $v_{C 1}$-coupled system $(\beta=15, \gamma=10)$. 


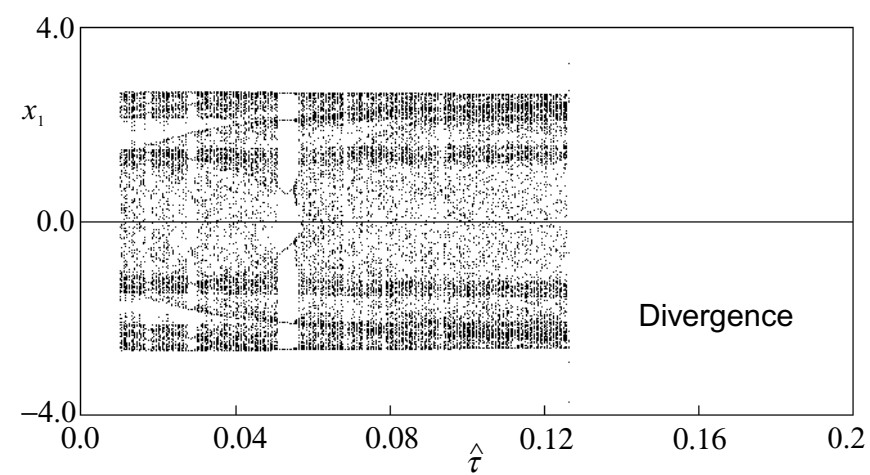

Figure 4: One parameter bifurcation diagram for $v_{C 2}$-coupled system $(\beta=18, \gamma=30)$.
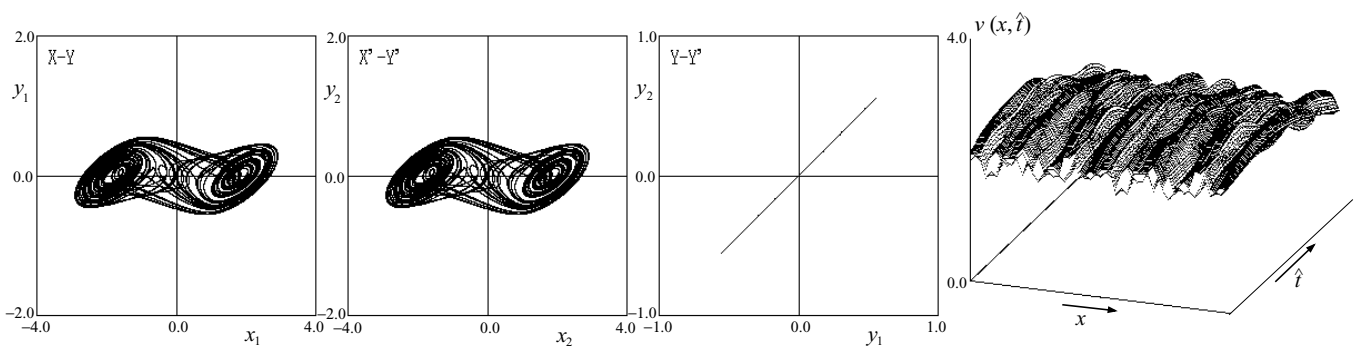

(a)
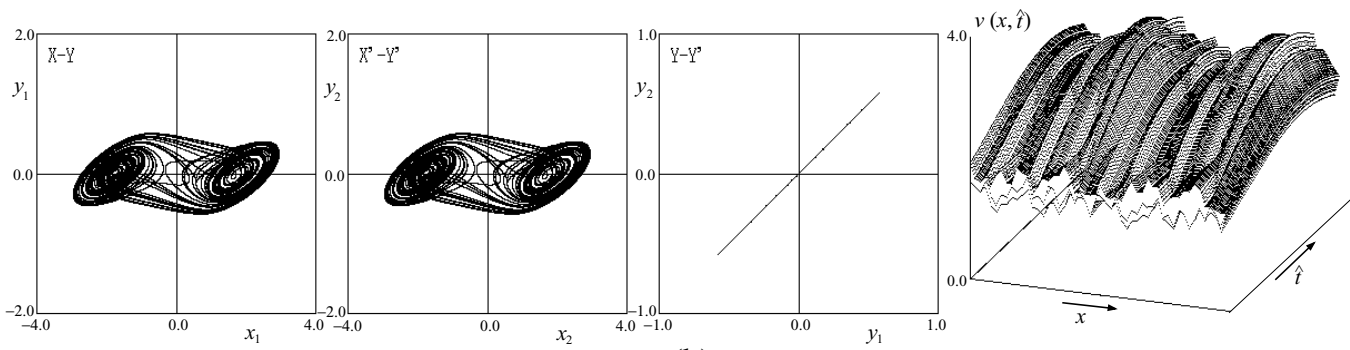

(b)

Figure 5: Voltage distribution for $v_{C 1}$-coupled system $(\beta=15)$. (a) $\gamma=10, \hat{\tau}=0.001$ (b) $\gamma=1, \hat{\tau}=0.01$.

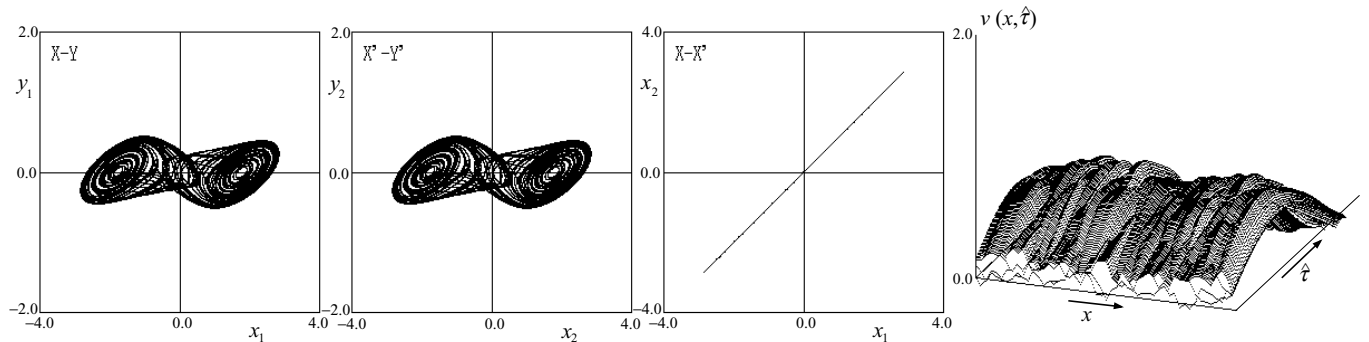

(a)
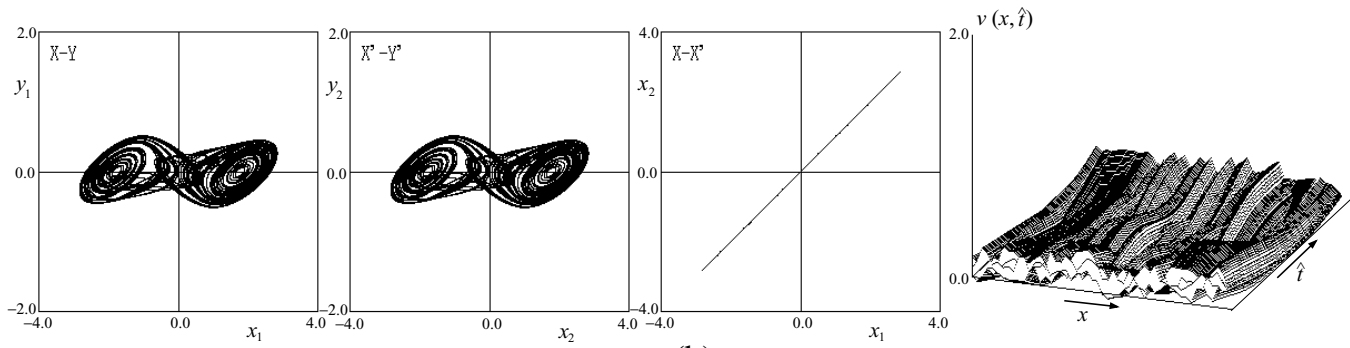

(b)

Figure 6: Voltage distribution for $v_{C 2}$-coupled system $(\beta=18$ ). (a) $\gamma=30, \hat{\tau}=0.01$ (b) $\gamma=15, \hat{\tau}=0.1$. 